\title{
THE PERIOPERATIVE FACTORS AFFECTING MORTALITY AND MORBIDITY IN PATIENTS UNDERGOING BILIARY SURGERIES
}

\author{
S. Senthilvel1 ${ }^{1}$ S. S. Abhinand ${ }^{2}$, Thangamani ${ }^{3}$ \\ ${ }^{1}$ Associate Professor, Department of General Surgery, Pudukkottai Medical College, Tamilnadu. \\ 2 Postgraduate Student, Department of General Surgery, KAPV Government Medical College, Trichy. \\ ${ }^{3}$ Postgraduate Student, Department of General Surgery, KAPV Government Medical College, Trichy.
}

ABSTRACT

\section{BACKGROUND}

Biliary tract surgeries are one of the most common surgeries done by general and laparoscopic surgeons, but mortality and morbidity among the patients undergoing these procedures remains high.

\section{MATERIALS AND METHODS}

This is a study of 150 cases of biliary tract disorders diagnosed and treated at KAPV Government Medical College and Mahatma Gandhi Memorial Government Hospital, Tiruchirapalli, Tamilnadu.

\section{RESULTS}

The morbidity and mortality remains high among patients with high bilirubin value, preoperative diabetes, raised renal parameters, absence of preoperative biliary drainage and longer duration of surgery.

\section{CONCLUSION}

The morbidity and mortality of the patients undergoing biliary surgeries, especially jaundiced patients can be controlled by preoperative biliary drainage, correcting renal parameters and choosing correct mode of surgical technique. Even then biliary tract surgeries are challenging to surgeons.

\section{KEYWORDS}

Biliary Tract Surgery, Jaundice, Morbidity and Mortality.

HOW TO CITE THIS ARTICLE: Senthilvel S, Abhinand SS, Thangamani. The perioperative factors affecting mortality and morbidity in patients undergoing biliary surgeries. J. Evolution Med. Dent. Sci. 2017;6(27):2236-2243, DOI: 10.14260/Jemds/2017/482

\section{BACKGROUND}

Biliary surgeries especially in patients with perioperative jaundice are challenging ${ }^{1}$ even though laparoscopic and endoscopic techniques have improved the visualisation of operative field. The modern investigations delineate the anatomy, but morbidity and mortality remains high for biliary surgeries.

This can be prevented to an extent by proper perioperative care and assessment. With proper assessment, some, if not all, of these factors can be controlled preoperatively leading to a better outcome of surgery. This study tries to identify certain preoperative risk factors in jaundiced patients who undergo surgery of the biliary tract and correlate them with postoperative mortality and morbidity. Around 18 preoperative factors are used to assess the outcome of biliary surgeries.

\section{MATERIALS AND METHODS}

\section{Data Collection}

This is a study of 150 Cases of biliary tract disease diagnosed and treated at KAPV Government Medical College and

Financial or Other, Competing Interest: None.

Submission 30-01-2016, Peer Review 16-03-2017,

Acceptance 22-03-2017, Published 03-04-2017.

Corresponding Author:

Dr.S.S. Abhinand,

JR Surgery,

KAPV Government Medical College,

Junior Resident Quarters L Block Room No 22,

Trichy- 620001

E-mail: abhinandss@gmail.com

DOI: $10.14260 /$ jemds $/ 2017 / 482$
Mahatma Gandhi Memorial Government Hospital, Tiruchirapalli, Tamilnadu for a period of 2 years.

\section{RESULTS}

\section{Research Design}

Hospital based cross sectional study.

\section{Inclusion Criteria}

Only those who consent to enrol in the study and those undergoing surgery in the biliary tract only, with a biliary drainage procedure.

\section{Exclusion Criteria}

Those who do not give consent and those undergoing surgery of biliary tract along with some other surgeries in the abdomen and those undergoing emergency biliary surgery following trauma. Cases with surgeries on biliary tract without a biliary drainage procedure are also excluded.

\section{Method of Data Collection}

Hospital records, interviewing the patients and also from investigation reports and lab results.

\section{Statistical Analysis}

Prevalence of each risk factor is expressed in terms of frequencies and percentages. Morbidity and mortality also is expressed in a similar manner. The relation between the primary and secondary objectives was studied using the Chi square test, with the help of Software, SPSS. Binary logistic 
regression was done to find out factors independently predicting the occurrence of morbidity and mortality.

\section{Aims and Objectives}

1. Primary Objective- To find out the prevalence of preoperative risk factors present in patients undergoing biliary tract surgeries.

2. Secondary Objective- To find out the morbidity and mortality among patients undergoing biliary tract surgeries within a followup period of 30 days.

\section{Following Factors are Studied}

\begin{tabular}{|c|c|c|c|}
\hline & $\begin{array}{c}\text { Risk } \\
\text { Factors }\end{array}$ & $\begin{array}{c}\text { Normal/ } \\
\text { No Risk }\end{array}$ & At Risk \\
\hline 1 & AGE & $<60$ years & $>60$ years \\
\hline 2 & Disease type & Benign & Malignant \\
\hline 3 & Fever & Absent & Present \\
\hline 4 & Duration of Jaundice & $<3$ weeks & $>3$ weeks \\
\hline 5 & Amount of Bilirubin & $<10 \mathrm{mg} / \mathrm{dL}$ & $>10 \mathrm{mg} / \mathrm{dL}$ \\
\hline 6 & Hypertension & Absent & Present \\
\hline 7 & Diabetes mellitus & Absent & Present \\
\hline 8 & $\begin{array}{c}\text { Preoperative biliary } \\
\text { drainage }\end{array}$ & Not done & Done \\
\hline 9 & Haematocrit & $>30 \%$ & $<30 \%$ \\
\hline 10 & TLC & $<10,000 / \mathrm{mm}^{3}$ & $>10,000 / \mathrm{mm}^{3}$ \\
\hline 11 & PT-INR & $<1.5$ & $>1.5$ \\
\hline 12 & S. Urea & $<40 \mathrm{mg} / \mathrm{dL}$ & $>40 \mathrm{mg} / \mathrm{dL}$ \\
\hline 13 & S. Creatinine & $<1.5 \mathrm{mg} / \mathrm{dL}$ & $>1.5 \mathrm{mg} / \mathrm{dL}$ \\
\hline 14 & AST & $<100 \mathrm{IU} / \mathrm{L}$ & $>100 \mathrm{IU} / \mathrm{L}$ \\
\hline 15 & ALT & $<100 \mathrm{IU} / \mathrm{L}$ & $>100 \mathrm{IU} / \mathrm{L}$ \\
\hline 16 & ALP & $<100 \mathrm{IU} / \mathrm{L}$ & $>150 \mathrm{IU} / \mathrm{L}$ \\
\hline 17 & HBV/HCV infection & Absent & Present \\
\hline 18 & Ascites & Absent & Present \\
\hline 19 & BMI & $17.5-24$ & $<17.5$ \\
\hline 20 & Operation time & $<3$ hours & $>3$ hours \\
\hline 21 & Type of surgery & Type 1 & Type 3 \\
\hline
\end{tabular}

\section{RESULTS}

\section{Interpretation}

Out of the 150 patients studied, 80 (53.3\%) were male and 70 (46.7.1\%) were female. $42.81 \%$ had benign and $57.19 \%$ had malignant cause for obstruction. $32.5 \%$ had fever in the preoperative period and $63.1 \%$ had jaundice for more than 3 weeks. $40 \%$ of patients had a serum bilirubin level more than $10 \mathrm{mg} / \mathrm{dL} .15 \%$ had systemic hypertension and 30\% had diabetes mellitus. $31.24 \%$ of patients underwent preoperative biliary drainage by endoscopic routes. $20.2 \%$ had a haematocrit less than 30 and $30.2 \%$ had a total leucocyte count more than $10,000 / \mathrm{mm}^{3}$. PT- INR value was more than 1.5 in $10.1 \% .11 \%$ of patients had a blood urea value more than $40 \mathrm{mg} / \mathrm{dL}$ and $5.2 \%$ had serum creatinine levels higher than $1.5 \mathrm{mg} / \mathrm{dL}$ in the preoperative period. AST and ALT elevation above $100 \mathrm{IU} / \mathrm{L}$ were observed in 34\% and $29.8 \%$ of patients respectively. Alkaline phosphatase levels in serum was elevated above $150 \mathrm{IU} / \mathrm{L}$ in $80.2 \%$ of patients. $33.5 \%$ had a BMI less than 17.5 and $14.9 \%$ had ascites. $66 \%$ of the surgeries took more than three hours to complete. $13 \%$ of the patients had postoperative renal failure. Surgical site infection occurred in $34 \%$ of patients. $9.6 \%$ had gastrointestinal haemorrhage and $7.2 \%$ patients developed intra-abdominal abscess. Infection and sepsis was observed in $20 \%$ and pulmonary complication in $25.6 \%$. The overall mortality observed is $9.4 \%$.

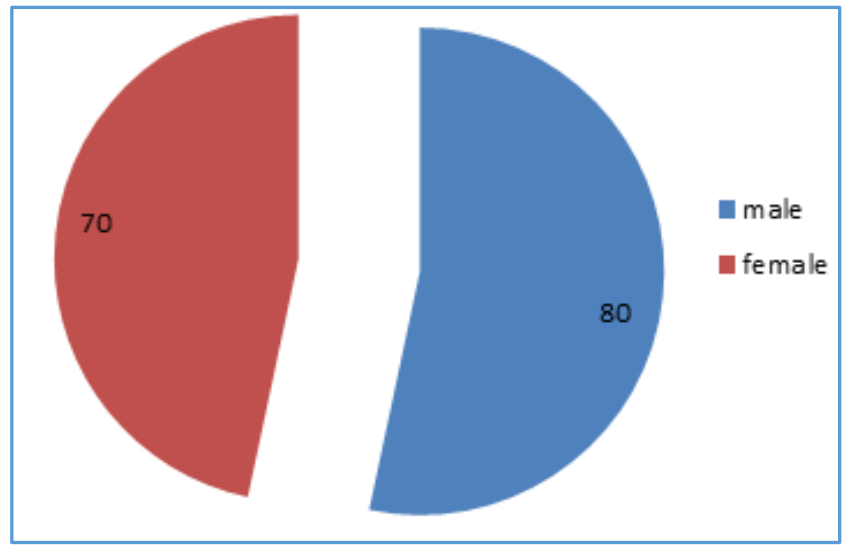

Figure 1

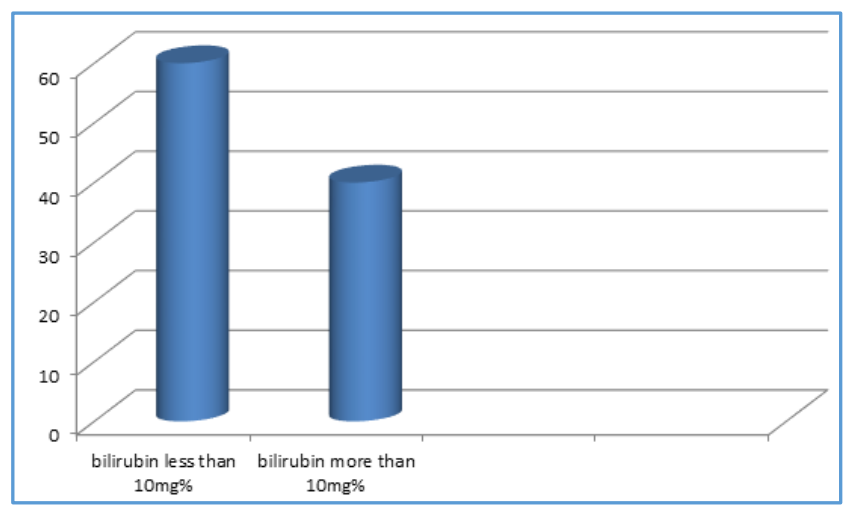

Figure 2

\begin{tabular}{|c|c|c|c|}
\hline & Patients' Frequency & \% Percent & Cumulative Percentage \\
\hline Absent & 127 & 85.0 & 85.0 \\
\hline Present & 23 & 15.0 & 100.0 \\
\hline Total & $\mathbf{1 5 0}$ & $\mathbf{1 0 0 . 0}$ & \\
\hline \multicolumn{2}{|c|}{ Table 2. Frequency and Percentage of Patients with Systemic Hypertension } \\
\hline
\end{tabular}

\begin{tabular}{|c|c|c|c|}
\hline & Frequency & \% Percent & Cumulative Percentage \\
\hline Absent & 105 & 70.0 & 70.0 \\
\hline Present & 45 & 30.0 & 30 \\
\hline Total & 150 & 100.0 & 100.0 \\
\hline \multicolumn{3}{|c|}{ Table 3. Diabetes } \\
\hline
\end{tabular}

Frequency and

percentage

of

patients

with

diabetes

mellitus 


\section{Type of Surgery}

- Drainage procedure alone- $19 \%$.

- Drainage procedure plus biliary enteric anastomosis$41 \%$.

- Drainage plus biliary enteric anastomosis plus liver/biliary resection.

- $\quad 40 \%$ Morbidity.

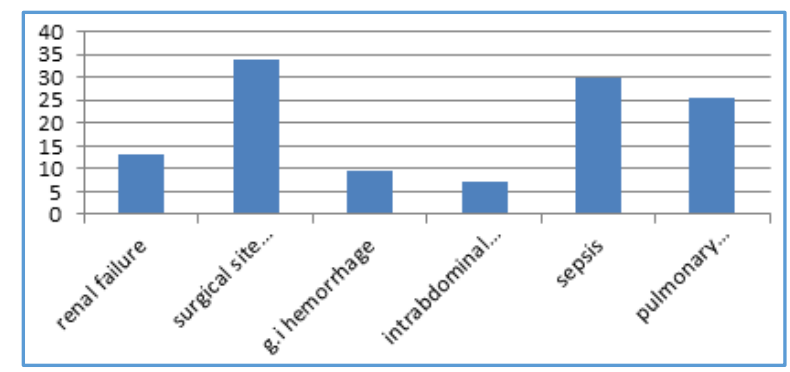

Figure 3

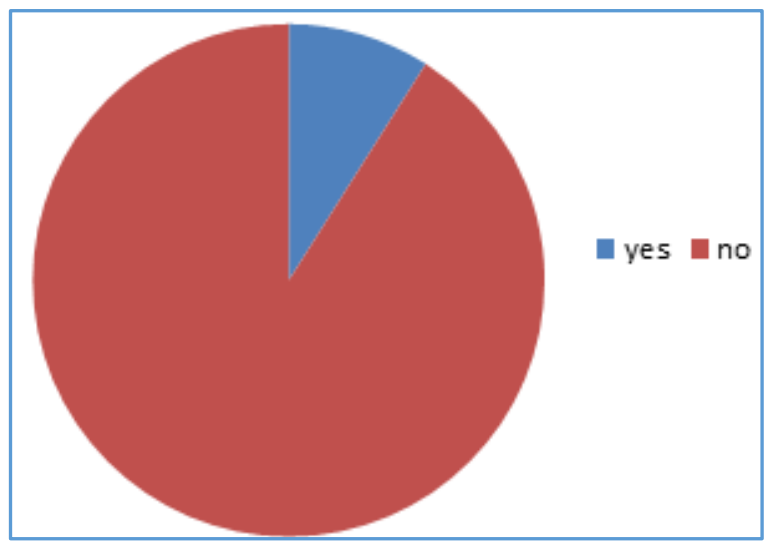

Figure 4

\section{Bivariate Analysis}

Bivariate analysis was done using Chi squared test to establish the effect (if any) of each risk factor on the outcome variables (morbidity and mortality). The statistical significance of the effect of each factor on outcome can be determined by the Pearson Chi square value. A value less than 0.05 is taken as statistically significant. Contingency tables were generated between each of the risk factors and outcome variables (morbidity and mortality). Pearson Chi squared test and Fisher's exact test (where numbers were small) were done. Odds ratio was also calculated. The following tables show the statistical significance of association between morbidity/mortality and the risk factors evaluated.

\section{Morbidity}

\begin{tabular}{|c|c|c|c|c|c|}
\hline & $\begin{array}{c}\text { Risk } \\
\text { Factors }\end{array}$ & $\begin{array}{c}\text { Pearson } \\
\text { Chi Square }\end{array}$ & df & P Value & Significance \\
\hline 1 & $\begin{array}{c}\text { Age more } \\
\text { than 60 years }\end{array}$ & 0.527 & 1 & 0.487 & \\
\hline 2 & $\begin{array}{c}\text { Malignant } \\
\text { disease }\end{array}$ & 6.957 & 1 & 0.005 & Significant \\
\hline 3 & $\begin{array}{c}\text { Type of } \\
\text { surgery }\end{array}$ & 1.906 & 2 & 0.312 & \\
\hline 4 & $\begin{array}{c}\text { Body mass } \\
\text { index less than } \\
17.5\end{array}$ & 0.701 & 1 & 0.297 & \\
\hline \multicolumn{7}{|c|}{ Table 4. Factors Affecting Renal Failure, Results of Chi- } \\
squared test
\end{tabular}

\begin{tabular}{|c|c|c|c|c|c|}
\hline & $\begin{array}{c}\text { Risk } \\
\text { Factors }\end{array}$ & $\begin{array}{c}\text { Pearson } \\
\text { Chi } \\
\text { Square }\end{array}$ & df & $\begin{array}{c}P \\
\text { value }\end{array}$ & Significance \\
\hline 1 & $\begin{array}{c}\text { Presence of } \\
\text { systemic } \\
\text { hypertension }\end{array}$ & 0.077 & 1 & 0.678 & \\
\hline 2 & $\begin{array}{l}\text { Presence of } \\
\text { diabetes } \\
\text { mellitus }\end{array}$ & 5.176 & 1 & 0.024 & Significant \\
\hline 3 & Presence of fever & 0.019 & 1 & 0.763 & \\
\hline
\end{tabular}

\begin{tabular}{|c|c|c|c|c|c|}
\hline & $\begin{array}{c}\text { Risk } \\
\text { Factors }\end{array}$ & $\begin{array}{c}\text { Pearson } \\
\text { Chi } \\
\text { Square }\end{array}$ & df & $\begin{array}{c}\text { P } \\
\text { value }\end{array}$ & Significance \\
\hline 1 & $\begin{array}{c}\text { Haematocrit } \\
\text { less than } 30 \%\end{array}$ & 4.278 & 1 & 0.018 & Significant \\
\hline 2 & $\begin{array}{c}\text { T L C more } \\
\text { than 10,000 }\end{array}$ & 4.780 & 1 & 0.022 & Significant \\
\hline 3 & $\begin{array}{c}\text { PT-INR more } \\
\text { than 1.5 }\end{array}$ & 0.074 & 1 & 0.692 & \\
\hline 4 & $\begin{array}{c}\text { S. Creatinine } \\
\text { more than 1.5 }\end{array}$ & 2.943 & 1 & 0.069 & \\
\hline 5 & $\begin{array}{c}\text { S. Urea more } \\
\text { than 40 mg\% }\end{array}$ & 4.241 & 1 & 0.023 & Significant \\
\hline Table 7. Factors Affecting Renal Failure, Results of Chi- \\
squared test
\end{tabular}

\begin{tabular}{|c|c|c|c|c|l|}
\hline & $\begin{array}{c}\text { Risk } \\
\text { Factors }\end{array}$ & $\begin{array}{c}\text { Pearson } \\
\text { Chi } \\
\text { Square }\end{array}$ & df & $\begin{array}{c}\text { P } \\
\text { value }\end{array}$ & Significance \\
\hline 1 & $\begin{array}{c}\text { AST more than } \\
100 \text { IU/L }\end{array}$ & 1.071 & 1 & 0.301 & \\
\hline 2 & $\begin{array}{c}\text { ALT more than } \\
100 \text { IU/L }\end{array}$ & 0.122 & 1 & 0.727 & Significant \\
\hline 3 & $\begin{array}{c}\text { ALP more than } \\
150 \text { IU/L }\end{array}$ & 4.583 & 1 & 0.032 & Tactors Affecting Renal Failure, Results of Chi- \\
squared test \\
\hline \multicolumn{7}{|c|}{ Table 8. Factors } \\
\hline \multicolumn{6}{|c|}{} \\
\hline
\end{tabular}

\begin{tabular}{|c|c|c|c|c|c|}
\hline & $\begin{array}{c}\text { Risk } \\
\text { Factors }\end{array}$ & \begin{tabular}{|c|} 
Pearson \\
Chi \\
Square
\end{tabular} & df & $\begin{array}{c}P \\
\text { value }\end{array}$ & Significance \\
\hline 1 & $\begin{array}{c}\text { Presence of } \\
\mathrm{HBV} / \mathrm{HCV} \\
\text { infection } \\
\end{array}$ & 0.436 & 1 & 0.502 & \\
\hline 2 & $\begin{array}{c}\text { Presence of } \\
\text { ascites }\end{array}$ & 2.735 & 1 & 0.077 & \\
\hline 3 & $\begin{array}{c}\text { Operation time } \\
\text { more than } 3 \\
\text { hours }\end{array}$ & 5.108 & 1 & 0.014 & Significant \\
\hline
\end{tabular}

From Tables 4 - 9, according to Pearson Chi-square test, the factors significantly affecting the development of renal failure are more than $10 \mathrm{mg} / \mathrm{dL}$ bilirubin level, duration of jaundice more than 3 weeks, malignant disease, diabetes mellitus, operation time more than 3 hours, high total leucocyte count, low haematocrit, elevated creatinine and a high alkaline phosphatase level. Binary logistic regression was done to identify risk factors which are independent predictors of post-operative renal failure. 
Pre-operative bilirubin levels more than $10 \mathrm{mg} / \mathrm{dL}$ was the only significant variable which independently predicted renal failure. None of the other factors had any significant independent association

\begin{tabular}{|c|c|c|c|c|l|}
\hline & $\begin{array}{c}\text { Risk } \\
\text { Factors }\end{array}$ & $\begin{array}{c}\text { Pearson } \\
\text { Chi } \\
\text { square }\end{array}$ & Df & $\begin{array}{c}\text { P } \\
\text { value }\end{array}$ & Significance \\
\hline 1 & $\begin{array}{c}\text { Age more } \\
\text { than 60 years }\end{array}$ & 0.281 & 1 & 0.543 & \\
\hline 2 & $\begin{array}{c}\text { Malignant } \\
\text { disease }\end{array}$ & 0.207 & 1 & 0.634 & \\
\hline 3 & $\begin{array}{c}\text { Type of } \\
\text { surgery }\end{array}$ & 6.241 & 1 & 0.014 & Significant \\
\hline 4 & $\begin{array}{c}\text { Body mass } \\
\text { index less } \\
\text { than 17.5 }\end{array}$ & 0.429 & 2 & 0.715 & \\
\hline \multicolumn{6}{|c|}{ Table 10. Factors Affecting Surgical Site Infection, } \\
Results of Chi-squared test
\end{tabular}

\begin{tabular}{|c|c|c|c|c|c|}
\hline & Risk factors & $\begin{array}{c}\text { Pearson } \\
\text { Chi } \\
\text { square }\end{array}$ & Df & $\begin{array}{c}P \\
\text { value }\end{array}$ & Significance \\
\hline 1 & $\begin{array}{c}\text { Presence of } \\
\text { systemic } \\
\text { hypertension } \\
\end{array}$ & 1.730 & 1 & 0.154 & \\
\hline 2 & $\begin{array}{c}\text { Presence of } \\
\text { diabetes } \\
\text { mellitus } \\
\end{array}$ & 3.546 & 1 & 0.029 & Significant \\
\hline 3 & $\begin{array}{l}\text { Presence of } \\
\text { fever }\end{array}$ & 0.394 & 1 & 0.328 & \\
\hline
\end{tabular}

\begin{tabular}{|c|c|c|c|c|c|}
\hline & Risk Factors & $\begin{array}{c}\text { Pearson } \\
\text { Chi } \\
\text { square }\end{array}$ & df & $\begin{array}{c}P \\
\text { value }\end{array}$ & Significance \\
\hline 1 & $\begin{array}{l}\text { Duration of } \\
\text { Jaundice } \\
\text { more than } 3 \\
\text { months }\end{array}$ & 2.920 & 1 & 0.140 & \\
\hline 2 & $\begin{array}{c}\text { Amount of } \\
\text { Bilirubin more } \\
\text { than } 10 \mathrm{mg} / \mathrm{dL}\end{array}$ & 3.872 & 1 & 0.039 & Significant \\
\hline 3 & $\begin{array}{c}\text { Preoperative } \\
\text { biliary drainage } \\
\text { done }\end{array}$ & 0.204 & 1 & 0.577 & \\
\hline
\end{tabular}

Table 12. Factors Affecting Surgical Site Infection, Results of Chi-squared test

\begin{tabular}{|c|c|c|c|c|l|}
\hline & $\begin{array}{c}\text { Risk } \\
\text { Factors }\end{array}$ & $\begin{array}{c}\text { Pearson } \\
\text { Chi Square }\end{array}$ & Df & $\begin{array}{c}\text { P } \\
\text { Value }\end{array}$ & Significance \\
\hline 1 & $\begin{array}{c}\text { Haematocrit } \\
\text { less than } 30 \%\end{array}$ & 1.993 & 1 & 0.174 & \\
\hline 2 & $\begin{array}{c}\text { T L C more } \\
\text { than 10,000 }\end{array}$ & 10.847 & 1 & 0.001 & Significant \\
\hline 3 & $\begin{array}{c}\text { PT-INR more } \\
\text { than 1.5 }\end{array}$ & 0.037 & 1 & 0.895 & Significant \\
\hline 4 & $\begin{array}{c}\text { S. Urea more } \\
\text { than 40 mg\% }\end{array}$ & 3.959 & 1 & 0.036 & 1 \\
\hline 5 & $\begin{array}{c}\text { S. Creatinine } \\
\text { more than 1.5 }\end{array}$ & 1.974 & 0.181 & \\
\hline \multicolumn{7}{|c|}{ Table 13. Factors Affecting Surgical Site Infection, Results } \\
of Chi-squared test
\end{tabular}

\begin{tabular}{|c|c|c|c|c|c|}
\hline & $\begin{array}{c}\text { Risk } \\
\text { Factors }\end{array}$ & $\begin{array}{c}\text { Pearson } \\
\text { Chi } \\
\text { square }\end{array}$ & df & $\begin{array}{c}\text { P } \\
\text { value }\end{array}$ & Significance \\
\hline 1 & $\begin{array}{c}\text { AST more } \\
\text { than 100 } \\
\text { IU/L }\end{array}$ & 2.769 & 1 & 0.076 & \\
\hline 2 & $\begin{array}{c}\text { ALT more } \\
\text { than 100 } \\
\text { IU/L }\end{array}$ & 3.247 & 1 & 0.049 & Significant \\
\hline 3 & $\begin{array}{c}\text { ALP more } \\
\text { than 150 } \\
\text { IU/L }\end{array}$ & 6.845 & 1 & 0.008 & Significant \\
\hline \multicolumn{6}{|c|}{ Table 14. Factors Affecting Surgical Site Infection, } \\
\hline \multicolumn{6}{|c|}{ Results of Chi-squared test } \\
\hline
\end{tabular}

\begin{tabular}{|c|c|c|c|c|c|}
\hline & $\begin{array}{c}\text { Risk } \\
\text { Factors }\end{array}$ & $\begin{array}{c}\text { Pearson } \\
\text { Chi } \\
\text { Square }\end{array}$ & df & $\begin{array}{c}\text { P } \\
\text { value }\end{array}$ & Significance \\
\hline 1 & $\begin{array}{c}\text { Presence of } \\
\text { HBV/HCV } \\
\text { infection }\end{array}$ & 0.482 & 1 & 0.480 & \\
\hline 2 & $\begin{array}{c}\text { Presence of } \\
\text { ascites }\end{array}$ & 5.259 & 1 & 0.022 & Significant \\
\hline 3 & $\begin{array}{c}\text { Operation } \\
\text { time more } \\
\text { than 3 hours }\end{array}$ & 1.270 & 1 & 0.260 & \\
\hline \multicolumn{5}{|c|}{ Table 15. Factors Affecting Surgical Site Infection, } \\
Results of Chi-squared test
\end{tabular}

From the results of Pearson Chi-squared test, it can be interpreted that the factors significantly affecting the occurrence of surgical site infections are alkaline phosphate levels above $150 \mathrm{IU} / \mathrm{L}$, body mass index less than 17.5, WBC count more than 10000 , presence of ascites, serum urea levels higher than $40 \mathrm{mg} / \mathrm{dL}$, presence of diabetes mellitus, amount of bilirubin more than $10 \mathrm{mg} / \mathrm{dL}$ and ALT levels more than $100 \mathrm{IU} / \mathrm{L}$.

Binary logistic regression identified a single significant independent predictor of surgical site infection, preoperative bilirubin levels more than $10 \mathrm{mg} / \mathrm{dL}$. None of the other factors were independently associated with the development of surgical site infection.

Factors Affecting Gastrointestinal Haemorrhage, Results of Chi-Squared Test

\begin{tabular}{|c|c|c|c|c|l|}
\hline & $\begin{array}{c}\text { Risk } \\
\text { Factors }\end{array}$ & $\begin{array}{c}\text { Pearson } \\
\text { Chi } \\
\text { square }\end{array}$ & df & $\begin{array}{c}\text { P } \\
\text { value }\end{array}$ & Significance \\
\hline 1 & $\begin{array}{c}\text { Age more } \\
\text { than } 60 \\
\text { years }\end{array}$ & 1.081 & 1 & 0.108 & \\
\hline 2 & $\begin{array}{c}\text { Malignant } \\
\text { disease }\end{array}$ & 1.002 & 1 & 0.292 & \\
\hline 3 & $\begin{array}{c}\text { Type of } \\
\text { surgery }\end{array}$ & 2.206 & 2 & 0.332 & \\
\hline 4 & $\begin{array}{c}\text { Body mass } \\
\text { index less } \\
\text { than 17.5 }\end{array}$ & 0.781 & 1 & 0.362 & \\
\hline Table 16. Factors Affecting Gastrointestinal Haemorrhage, \\
Results of Chi-squared test
\end{tabular}




\begin{tabular}{|c|c|c|c|c|c|}
\hline & Risk Factors & $\begin{array}{c}\text { Pearson } \\
\text { Chi } \\
\text { square }\end{array}$ & df & $\begin{array}{c}\text { P } \\
\text { value }\end{array}$ & Significance \\
\hline 1 & $\begin{array}{c}\text { Presence of } \\
\text { systemic } \\
\text { hypertension }\end{array}$ & 0.320 & 1 & 0.491 & \\
\hline 2 & $\begin{array}{c}\text { Presence of } \\
\text { diabetes mellitus }\end{array}$ & 0.049 & 1 & 0.781 & \\
\hline 3 & Presence of fever & 1.065 & 1 & 0.167 & \\
\hline Table 17. Factors Affecting Gastrointestinal Haemorrhage, \\
Results of Chi-squared test
\end{tabular}

\begin{tabular}{|c|c|c|c|c|c|}
\hline & $\begin{array}{c}\text { Risk } \\
\text { Factors }\end{array}$ & $\begin{array}{c}\text { Pearson } \\
\text { Chi } \\
\text { Square }\end{array}$ & df & $\begin{array}{c}\text { P } \\
\text { Value }\end{array}$ & Significance \\
\hline 1 & $\begin{array}{c}\text { Duration of } \\
\text { Jaundice more } \\
\text { than 3 months }\end{array}$ & 0.681 & 1 & 0.398 & \\
\hline 2 & $\begin{array}{c}\text { Amount of } \\
\text { Bilirubin more } \\
\text { than 10 mg/dL }\end{array}$ & 5.201 & 1 & 0.014 & Significant \\
\hline 3 & $\begin{array}{c}\text { Preoperative } \\
\text { biliary drainage } \\
\text { done }\end{array}$ & 1.23 & 1 & 0.247 & \\
\hline Table 18: Factors Affecting Gastrointestinal Haemorrhage, \\
Results of Chi-squared test
\end{tabular}

\begin{tabular}{|c|c|c|c|c|c|}
\hline & $\begin{array}{c}\text { Risk } \\
\text { Factors }\end{array}$ & $\begin{array}{c}\text { Pearson } \\
\text { Chi } \\
\text { Square }\end{array}$ & df & $\begin{array}{c}\text { P } \\
\text { value }\end{array}$ & Significance \\
\hline 1 & $\begin{array}{c}\text { Haematocrit } \\
\text { less than 30 \% }\end{array}$ & 0.587 & 1 & 0.391 & \\
\hline 2 & $\begin{array}{c}\text { T L C more } \\
\text { than 10,000 }\end{array}$ & 12.679 & 1 & 0.001 & Significant \\
\hline 3 & $\begin{array}{c}\text { PT-INR more } \\
\text { than 1.5 }\end{array}$ & 14.632 & 1 & 0.001 & Significant \\
\hline 4 & $\begin{array}{c}\text { S. Creatinine } \\
\text { more than 1.5 }\end{array}$ & 0.129 & 1 & 0.472 & \\
\hline 5 & $\begin{array}{c}\text { S. Urea more } \\
\text { than } 40 \text { mg\% }\end{array}$ & 0.385 & 1 & 0.535 & \\
\hline
\end{tabular}

Table 19. Factors Affecting Gastrointestinal Haemorrhage, Results of Chi-squared test

\begin{tabular}{|c|c|c|c|c|c|}
\hline & $\begin{array}{c}\text { Risk } \\
\text { Factors }\end{array}$ & $\begin{array}{c}\text { Pearson } \\
\text { Chi } \\
\text { Square }\end{array}$ & df & $\begin{array}{c}\mathbf{P} \\
\text { Value }\end{array}$ & Significance \\
\hline 1 & $\begin{array}{c}\text { AST more } \\
\text { than } 100 \\
\text { IU/L }\end{array}$ & 0.947 & 1 & 0.330 & \\
\hline 2 & $\begin{array}{c}\text { ALT more } \\
\text { than } 100 \\
\text { IU/L }\end{array}$ & 3.345 & 1 & 0.067 & \\
\hline 3 & $\begin{array}{c}\text { ALP more } \\
\text { than } 150 \\
\text { IU/L }\end{array}$ & 3.966 & 1 & 0.046 & Significant \\
\hline
\end{tabular}

Table 20. Factors Affecting Gastrointestinal Haemorrhage, Results of Chi-squared test

\begin{tabular}{|c|c|c|c|c|l|}
\hline 1 & $\begin{array}{c}\text { Risk } \\
\text { Factors }\end{array}$ & $\begin{array}{c}\text { Pearson } \\
\text { Chi } \\
\text { Square }\end{array}$ & df & $\begin{array}{c}\text { P } \\
\text { Value }\end{array}$ & Significance \\
\hline $\begin{array}{c}\text { Presence of } \\
\text { infection }\end{array}$ & 0.114 & 1 & 0.736 & \\
\hline 2 & $\begin{array}{c}\text { Presence of } \\
\text { ascites }\end{array}$ & 0.005 & 1 & 0.942 & \\
\hline 3 & $\begin{array}{c}\text { Operation time } \\
\text { more than 3 } \\
\text { hours }\end{array}$ & 1.256 & 1 & 0.262 & \\
\hline \multicolumn{5}{|c|}{$\begin{array}{c}\text { Table 21. Factors Affecting Gastrointestinal } \\
\text { Haemorrhage, Results of Chi-squared test }\end{array}$} \\
\hline
\end{tabular}

From the $\mathrm{P}$ - values in Chi-squared test, it can be interpreted that the factors associated with the occurrence of gastrointestinal haemorrhage are total leucocyte count more than $10,000 / \mathrm{mm}^{3}$, PT-INR more than 1.5 , amount of bilirubin more than $10 \mathrm{mg} / \mathrm{dL}$ and alkaline phosphatase level more than 150 IU.

Binary logistic regression was done and found that total leucocyte count above $10,000 / \mathrm{mm}^{3}$ and PT-INR above 1.5 are independently associated with the occurrence of gastrointestinal bleeding.

\begin{tabular}{|c|c|c|c|c|c|}
\hline & $\begin{array}{c}\text { Risk } \\
\text { Factors }\end{array}$ & $\begin{array}{c}\text { Pearson } \\
\text { Chi } \\
\text { Square }\end{array}$ & df & $\begin{array}{c}\text { P } \\
\text { value }\end{array}$ & Significance \\
\hline 1 & $\begin{array}{c}\text { Age more } \\
\text { than } 60 \\
\text { years }\end{array}$ & 0.327 & 1 & 0.519 & \\
\hline 2 & $\begin{array}{c}\text { Malignant } \\
\text { disease }\end{array}$ & 0.026 & 1 & 0.851 & \\
\hline 3 & $\begin{array}{c}\text { Type of } \\
\text { surgery }\end{array}$ & 0.968 & 2 & 0.436 & \\
\hline 4 & $\begin{array}{c}\text { Body mass } \\
\text { index less } \\
\text { than 17.5 }\end{array}$ & 0.144 & 1 & 0.544 & \\
\hline \multicolumn{7}{|c|}{ Table 22. Factors Affecting the Development of Intra- } \\
\hline
\end{tabular}

Abdominal Abscess, Results of Chi- squared test

\begin{tabular}{|c|c|c|c|c|c|}
\hline & $\begin{array}{c}\text { Risk } \\
\text { Factors }\end{array}$ & $\begin{array}{c}\text { Pearson } \\
\text { Chi } \\
\text { Square }\end{array}$ & df & $P$ value & Significance \\
\hline 1 & \begin{tabular}{|c|} 
Presence of \\
systemic \\
hypertension
\end{tabular} & 0.094 & 1 & 0.759 & \\
\hline 2 & $\begin{array}{c}\text { Presence of } \\
\text { diabetes } \\
\text { mellitus } \\
\end{array}$ & 1.343 & 1 & 0.246 & \\
\hline 3 & $\begin{array}{c}\text { Presence of } \\
\text { fever }\end{array}$ & 0.500 & 1 & 0.986 & \\
\hline
\end{tabular}

Abdominal Abscess, Results of Chi- squared test

\begin{tabular}{|c|c|c|c|c|c|}
\hline & $\begin{array}{c}\text { Risk } \\
\text { Factors }\end{array}$ & $\begin{array}{c}\text { Pearson } \\
\text { Chi square }\end{array}$ & df & $\begin{array}{c}\text { P } \\
\text { value }\end{array}$ & Significance \\
\hline 1 & $\begin{array}{c}\text { Duration of } \\
\text { Jaundice more } \\
\text { than 3 months }\end{array}$ & 0.279 & 1 & 0.597 & \\
\hline 2 & $\begin{array}{c}\text { Amount of } \\
\text { Bilirubin more } \\
\text { than 10 mg/dL }\end{array}$ & 0.146 & 1 & 0.702 & \\
\hline 3 & $\begin{array}{c}\text { Preoperative } \\
\text { biliary drainage } \\
\text { done }\end{array}$ & 0.278 & 1 & 0.598 & \\
\hline \multicolumn{3}{|c|}{ Table 24. Factors Affecting the Development of Intra- } \\
Abdominal Abscess, Results of Chi- squared test \\
\hline
\end{tabular}




\begin{tabular}{|c|c|c|c|c|l|}
\hline & $\begin{array}{c}\text { Risk } \\
\text { Factors }\end{array}$ & $\begin{array}{c}\text { Pearson } \\
\text { Chi } \\
\text { Square }\end{array}$ & df & $\begin{array}{c}\text { P } \\
\text { value }\end{array}$ & Significance \\
\hline 1 & $\begin{array}{c}\text { Haematocrit } \\
\text { less than 30\% }\end{array}$ & 1.451 & 1 & 0.228 & \\
\hline 2 & $\begin{array}{c}\text { T L C more } \\
\text { than 10,000 }\end{array}$ & 1.796 & 1 & 0.180 & \\
\hline 3 & $\begin{array}{c}\text { PT-INR more } \\
\text { than 1.5 }\end{array}$ & 1.045 & 1 & 0.307 & \\
\hline 4 & $\begin{array}{c}\text { S. Creatinine } \\
\text { more than 1.5 }\end{array}$ & 0.043 & 1 & 0.836 & \\
\hline 5 & $\begin{array}{c}\text { S. Urea more } \\
\text { than 40 mg\% }\end{array}$ & 2.870 & 1 & 0.090 & \\
\hline \multicolumn{6}{|c|}{ Table 25. Factors affecting the development of intra- } \\
abdominal abscess, results of Chi- squared test
\end{tabular}

\begin{tabular}{|c|c|c|c|c|l|}
\hline & $\begin{array}{c}\text { Risk } \\
\text { Factors }\end{array}$ & $\begin{array}{c}\text { Pearson } \\
\text { Chi } \\
\text { Square }\end{array}$ & df & $\begin{array}{c}\text { P } \\
\text { value }\end{array}$ & Significance \\
\hline 1 & $\begin{array}{c}\text { AST more } \\
\text { than } \\
100 \mathrm{IU} / \mathrm{L}\end{array}$ & 0.786 & 1 & 0.375 & \\
\hline 2 & $\begin{array}{c}\text { ALT more } \\
\text { than } \\
100 \mathrm{IU} / \mathrm{L}\end{array}$ & 2.299 & 1 & 0.135 & \\
\hline 3 & $\begin{array}{c}\text { ALP more } \\
\text { than } \\
150 \mathrm{IU} / \mathrm{L}\end{array}$ & 0.025 & 1 & 0.874 & \\
\hline \multicolumn{3}{|c|}{ Table 26.Factors Affecting the Development of Intra- } \\
Abdominal Abscess, Results of Chi- squared test
\end{tabular}

\begin{tabular}{|c|c|c|c|c|c|}
\hline & $\begin{array}{c}\text { Risk } \\
\text { Factors }\end{array}$ & $\begin{array}{c}\text { Pearson } \\
\text { Chi } \\
\text { Square }\end{array}$ & df & $\begin{array}{c}\text { P } \\
\text { value }\end{array}$ & Significance \\
\hline 1 & $\begin{array}{c}\text { Presence of } \\
\text { HBV/HCV } \\
\text { infection }\end{array}$ & 1.600 & 1 & 0.206 & \\
\hline 2 & $\begin{array}{c}\text { Presence of } \\
\text { ascites }\end{array}$ & 1.592 & 1 & 0.207 & \\
\hline 3 & $\begin{array}{c}\text { Operation } \\
\text { time more } \\
\text { than 3 hours }\end{array}$ & 0.797 & 1 & 0.372 & \\
\hline \multicolumn{3}{|c|}{ Table 27: Factors Affecting the Development of Intra- } \\
Abdominal Abscess, Results of Chi- squared test
\end{tabular}

From Tables 22 - 27, after performing Chi-squared test, none of the tested factors show any statistically significant association with the occurrence of intra-abdominal abscess.

Factors Affecting Infection and Sepsis, Results of ChiSquared Test

\begin{tabular}{|c|c|c|c|c|c|}
\hline & $\begin{array}{c}\text { Risk } \\
\text { Factors }\end{array}$ & $\begin{array}{c}\text { Pearson } \\
\text { Chi } \\
\text { Square }\end{array}$ & df & $\begin{array}{c}\text { P } \\
\text { value }\end{array}$ & Significance \\
\hline 1 & $\begin{array}{c}\text { Age more } \\
\text { than 60 years }\end{array}$ & 0.029 & 1 & 0.745 & \\
\hline 2 & $\begin{array}{c}\text { Malignant } \\
\text { disease }\end{array}$ & 5.965 & 1 & 0.009 & Significant \\
\hline 3 & $\begin{array}{c}\text { Type of } \\
\text { surgery }\end{array}$ & 7.980 & 2 & 0.012 & Significant \\
\hline 4 & $\begin{array}{c}\text { Body mass } \\
\text { index less } \\
\text { than 17.5 }\end{array}$ & 8.358 & 1 & 0.001 & Significant \\
\hline \multicolumn{7}{|c|}{ Table 28. Factors Affecting Infection and Sepsis, Results of } \\
Chi-squared test
\end{tabular}

\begin{tabular}{|c|c|c|c|c|c|}
\hline & $\begin{array}{c}\text { Risk } \\
\text { Factors }\end{array}$ & $\begin{array}{c}\text { Pearson } \\
\text { Chi } \\
\text { Square }\end{array}$ & df & $\begin{array}{c}\text { P } \\
\text { value }\end{array}$ & Significance \\
\hline 1 & $\begin{array}{c}\text { Presence of } \\
\text { systemic } \\
\text { hypertension }\end{array}$ & 4.404 & 1 & 0.019 & Significant \\
\hline 2 & $\begin{array}{c}\text { Presence of } \\
\text { diabetes } \\
\text { mellitus }\end{array}$ & 12.325 & 1 & 0.001 & Significant \\
\hline 3 & $\begin{array}{c}\text { Presence of } \\
\text { fever }\end{array}$ & 4.098 & 1 & 0.021 & Significant \\
\hline Table 29. Factors Affecting Infection and Sepsis, Results of \\
Chi-squared test
\end{tabular}

\begin{tabular}{|c|c|c|c|c|l|}
\hline & $\begin{array}{c}\text { Risk } \\
\text { Factors }\end{array}$ & $\begin{array}{c}\text { Pearson } \\
\text { Chi } \\
\text { Square }\end{array}$ & df & $\begin{array}{c}\text { P } \\
\text { value }\end{array}$ & Significance \\
\hline 1 & $\begin{array}{c}\text { Duration of } \\
\text { Jaundice more } \\
\text { than 3 months }\end{array}$ & 13.686 & 1 & 0.001 & Significant \\
\hline 2 & $\begin{array}{c}\text { Amount of } \\
\text { Bilirubin more } \\
\text { than 10 mg/dL }\end{array}$ & 5.257 & 1 & 0.011 & Significant \\
\hline 3 & $\begin{array}{c}\text { Preoperative } \\
\text { biliary drainage } \\
\text { done }\end{array}$ & 2.240 & 1 & 0.108 & \\
\hline
\end{tabular}

Table 30. Factors Affecting Infection and Sepsis, Results of Chi-squared test

\begin{tabular}{|c|c|c|c|c|l|}
\hline & $\begin{array}{c}\text { Risk } \\
\text { Factors }\end{array}$ & $\begin{array}{c}\text { Pearson } \\
\text { Chi } \\
\text { Square }\end{array}$ & df & $\begin{array}{c}\text { P } \\
\text { value }\end{array}$ & Significance \\
\hline 1 & $\begin{array}{c}\text { Haematocrit } \\
\text { less than 30\% }\end{array}$ & 3.412 & 1 & 0.046 & \\
\hline 2 & $\begin{array}{c}\text { T L C more } \\
\text { than 10,000 }\end{array}$ & 5.270 & 1 & 0.029 & Significant \\
\hline 3 & $\begin{array}{c}\text { PT-INR more } \\
\text { than 1.5 }\end{array}$ & 0.074 & 1 & 0.619 & \\
\hline 4 & $\begin{array}{c}\text { S. Creatinine } \\
\text { more than 1.5 }\end{array}$ & 3.306 & 1 & 0.051 & \\
\hline 5 & $\begin{array}{c}\text { S. Urea more } \\
\text { than 40 mg\% }\end{array}$ & 5.80 & 1 & 0.019 & Significant \\
\hline Table 31. Factors Affecting Infection and Sepsis, Results of \\
Chi-squared test
\end{tabular}

\begin{tabular}{|c|c|c|c|c|c|}
\hline & $\begin{array}{c}\text { Risk } \\
\text { Factors }\end{array}$ & $\begin{array}{l}\text { Pearson } \\
\text { Chi } \\
\text { Square }\end{array}$ & df & $\begin{array}{c}\mathbf{P} \\
\text { Value }\end{array}$ & Significance \\
\hline 1 & $\begin{array}{c}\text { Presence of } \\
\mathrm{HBV} / \mathrm{HCV} \\
\text { infection }\end{array}$ & 0.926 & 1 & 0.291 & \\
\hline 2 & $\begin{array}{l}\text { Presence of } \\
\text { ascites }\end{array}$ & 6.381 & 1 & 0.010 & Significant \\
\hline 3 & $\begin{array}{c}\text { Operation time } \\
\text { more than } 3 \\
\text { hours }\end{array}$ & 7.226 & 1 & 0.006 & Significant \\
\hline
\end{tabular}

From Tables 28-32, according to Pearson Chi-squared test, the factors significantly affecting infective and septic complications are total leucocyte count more than 10000, amount of bilirubin more than $10 \mathrm{mg} / \mathrm{dL}$, body mass index less than 17.5, an operation time more than 3 hours, presence of diabetes mellitus, alkaline phosphatase levels 
above $150 \mathrm{IU}$, the type of surgical procedure, fever, serum creatinine levels higher than 1.5, malignant cause for biliary obstruction, duration of jaundice more than 3 weeks. After applying binary logistic regression, the factors found to have independent association with infective and septic complications are duration of jaundice more than three weeks, diabetes mellitus, elevated serum creatinine value and the type of surgery done.

\section{RESULTS}

\section{In our study, it has been interpreted that}

1. Renal failure is more common in patients with malignancy, diabetes mellitus, low haematocrit, total leucocyte count more than 10000 , urea $>40$, ALP $>150$.

2. Surgical site infection varies with type of surgery and its duration, bilirubin $>10 \mathrm{mg} \%$, urea $>40 \mathrm{mg} \%$.

3. Sepsis is more commonly seen in patients with malignant condition, patients with BMI less than 17.5, systemic hypertension, diabetes mellitus, fever, total leucocyte count $>10000$, urea $>40 \mathrm{mg} \%$, alkaline phosphatase $>150$, with ascites, duration of surgery $>3$ hrs.

\section{DISCUSSION}

Prevalence of each risk factor in the study population is expressed and compared with morbidity and mortality in postoperative period. Most of the risk factors are nonmodifiable. Certain modifiable factors include a low haematocrit and BMI. These can be addressed by giving extra care to the nutrition of the patient in the preoperative period. Even total parenteral nutrition is justified in selected patients, since the concerns regarding mucosal atrophy and bacterial translocation limited its use. Nutritional problems can also be due to anomalous hepatic and biliary dysfunction which should also be cared off.

Renal failure remains an important postoperative problem in surgeries for biliary obstruction. ${ }^{2}$ The percentage of renal failure in current study is $10 \%$. Single most important factor with independent effect on renal failure identified in present study is a preoperative bilirubin level more than $10 \mathrm{mg} / \mathrm{dL}^{3}$. Thus, preoperative biliary drainage may play a significant role in decreasing postoperative renal failure as well. ${ }^{4}$ But here again, careful patient selection is warranted.

The incidence of surgical site in percentage in the present study is 30 . The single most important independent predictor of surgical site infection identified by regression analysis is an amount of bilirubin more than $10 \mathrm{mg} / \mathrm{dL} .^{5}$ Measures to reduce pre-operative bilirubin values to less than ten percentage should be taken if this potentially serious complication is to be prevented.

Incidence of infection and sepsis in present study is $20 \%$, which is in excess compared to other surgeries and in literature, probably because of the small sample size and in part because of the late presentation of most of our patients to the surgeon. Independent predictors of sepsis identified in regression analysis are duration of jaundice more than three weeks, diabetes mellitus, elevated serum creatinine value and the type of surgery done. ${ }^{6}$ Sepsis is the only complication in the present study which has association with the type of surgery.
Prevention of this complication includes prompt biliary drainage in selected patients, control of infections and reduction of hepatic ascites by medical management. Mortality rates varying between 8 and $33 \%$ had been reported in literature for surgeries to relieve biliary obstruction. Mortality rate observed in the present study is 9.4\% which is comparable to older studies.(1) The malignant causes for biliary obstruction, ${ }^{7}$ duration of jaundice more than 3 weeks, ${ }^{8}$ amount of bilirubin more than $10 \mathrm{mg} / \mathrm{dL}^{9}$ presence of diabetes mellitus, total leucocyte count more than 10000 , serum creatinine levels higher than 1.5 , alkaline phosphate levels above $150 \mathrm{IU} / \mathrm{L}$, body mass index less than 17.5 and an operation time more than 3 hours were all found significant in the bivariate analysis.

But while taking the inter-relationship of these factors into account and after performing a regression analysis, it was found that operating time more than 3 hours, elevated total leucocyte count (more than 10,000/mm3) and a bilirubin level more than $10 \mathrm{mg} / \mathrm{dL}$ are independently associated with a high mortality rate.

\section{Summary}

In this hospital based cross sectional study, conducted in a tertiary care centre, over a period of 24 months, a total of 150 cases of biliary obstructions were studied. All of them underwent surgery to relieve biliary obstruction. The prevalence of 21 different risk factors in this study population was identified. The relationship between these 21 clinical, laboratory, and operative parameters to postoperative morbidity and mortality was analysed. Prevalence of the proposed risk factors are expressed in percentage. The relationship of preoperative factors and postoperative complications were analysed using Chisquared tests and Fisher's exact test where numbers were small. Binary logistic regression was used to test the relationship between the preoperative factors and postoperative mortality. The overall mortality observed is 9.4\%. After performing Chi-square tests and binary logistic regression, the factors with independent effect on morbidity and mortality were listed. An operating time more than 3 hours, ${ }^{10}$ elevated total leucocyte count (more than $10,000 / \mathrm{mm} 3)^{11}$ and a bilirubin level more than $10 \mathrm{mg} / \mathrm{dL}$ are independently associated with a high mortality rate. Preoperative bilirubin levels of more than $10 \mathrm{mg} / \mathrm{dL}$ is independently associated with higher incidence of postoperative renal failure and surgical site infection.

Duration of jaundice more than three weeks, ${ }^{12}$ diabetes mellitus, ${ }^{13}$ elevated serum creatinine value above $1.5 \mathrm{mg} \%{ }^{14}$ and the type of surgery done are the independent factors affecting post-operative sepsis. None of the tested variables showed any statistically significant relation with the development of intra-abdominal abscess formation.

\section{CONCLUSION}

The morbidity and mortality of the patients undergoing biliary surgeries especially jaundiced patients can be controlled by preoperative biliary drainage, correcting renal parameters and choosing correct mode of surgical technique. Even then biliary tract surgeries are challenging to surgeons. 


\section{REFERENCES}

[1] Cherqui D, Benoist S, Malassagne B, et al. Major liver resection for carcinoma in jaundiced patients without preoperative biliary drainage. Arch Surg 2000;135(3):302-8.

[2] Dixon JM, Armstrong CP, Duffy SW, et al. Factors affecting morbidity and mortality after surgery for obstructive jaundice: a review of 373 patients. Gut1983;24(9):845-52.

[3] de Mendonça A, Vincent JL, Suter PM, et al. Acute renal failure in the ICU: risk factors and outcome evaluated by the SOFA score. Intensive Care Medicine 2000;26(7):915-21.

[4] Poon RT, Fan ST, Lo CM, et al. Improving perioperative outcome expands the role of hepatectomy in management of benign and malignant hepatobiliary diseases: analysis of 1222 consecutive patients from a prospective database. Ann Surg 2004;240(4):698-708.

[5] Collins LA, Samore MH, Roberts MS, et al. Risk factors for invasive fungal infections complicating orthotopic liver transplantation. J Infect Dis1994;170(3):644-52.

[6] Pérez AJA, González JJ, Baldonedo RF, et al.Clinical course, treatment and multivariate analysis of risk factors for pyogenic liver abscess. Am JSurg 2001;181(2):177-86.

[7] Schmassmann A, von Gunten E, Knuchel J, et al. Wallstents versus plastic stents in malignant biliary obstruction: effects of stent patency of the first and second stent on patient compliance and survival. Am J Gastroenterol1996;91(4):654-9.
[8] Burris HA, Moore MJ, Andersen J, et al. Improvements in survival and clinical benefit with gemcitabine as first-line therapy for patients with advanced pancreas cancer: a randomized trial. J clin oncol 1997;15(6):2403-13.

[9] Goris RJ, te Boekhorst TP, Nuytinck JK, et al. Multipleorgan failure: generalized autodestructive inflammation? Arch Surg 1985;120(10):1109-15.

[10] Trede MI, Schwall G, Saeger HD. Survival after pancreaticoduodenectomy. 118 consecutive resections without an operative mortality. Ann Surg 1990;211(4):447-58.

[11] Pitt HA, Cameron JL, Postier RG, et al. Factors affecting mortality in biliary tract surgery. Am J Surg 1981;141(1):66-72.

[12] Maisels MJ, Kring E. Length of stay, jaundice and hospital readmission. Pediatrics 1998;101(6):995-8.

[13] Pories WJ, Swanson MS, MacDonald KG, et al. Who would have thought it? An operation proves to be the most effective therapy for adult-onset diabetes mellitus. AnnSurg 1995;222(3):339-52.

[14] Gallardo LM, Gutierrez HME, Pérez SG, et al. Risk factors for renal dysfunction in the postoperative course of liver transplant. Liver transpl 2004;10(11):1379-85. 